\title{
Dynamical cluster and hypernuclei production in heavy-ion collisions
}

\author{
Susanne Gläßel ${ }^{1}$, Viktar Kireyeu ${ }^{2}$, Vadim Voronyuk $^{2}$, Jörg Aichelin $^{3,4}$, Christoph Blume ${ }^{1}$, \\ Elena Bratkovskaya ${ }^{5,6,7, *}$, Gabriele Coci $^{5}$, Vadim Kolesnikov ${ }^{2}$, and Michael Winn ${ }^{3}$ \\ ${ }^{1}$ Institut für Kernphysik, Johann Wolfgang Goethe-Universität, Max-von-Laue-Str. 1, 60438 Frankfurt \\ am Main, Germany \\ ${ }^{2}$ Joint Institute for Nuclear Research, Joliot-Curie 6, 141980 Dubna, Moscow region, Russia \\ ${ }^{3}$ SUBATECH, Université de Nantes, IMT Atlantique, IN2P3/CNRS, 4 rue Alfred Kastler, 44307 Nantes \\ cedex 3, France \\ ${ }^{4}$ Frankfurt Institute for Advanced Studies, Ruth Moufang Str. 1, 60438 Frankfurt, Germany \\ ${ }^{5}$ GSI Helmholtzzentrum für Schwerionenforschung GmbH, Planckstr. 1, 64291 Darmstadt, Germany \\ ${ }^{6}$ Institut für Theoretische Physik, Johann Wolfgang Goethe-Universität, Max-von-Laue-Str. 1, 60438 \\ Frankfurt am Main, Germany \\ ${ }^{7}$ Helmholtz Research Academy Hesse for FAIR (HFHF), GSI Helmholtz Center for Heavy Ion Physics, \\ Campus Frankfurt, 60438 Frankfurt, Germany
}

\begin{abstract}
We study light cluster and hypernuclei production in heavy-ion collisions from SIS to RHIC energies based on the n-body dynamical transport approach PHQMD (Parton-Hadron-Quantum-Molecular-Dynamics). In PHQMD clusters are formed dynamically due to the interactions between baryons described on the basis of Quantum Molecular Dynamics (QMD) which allows to propagate the n-body Wigner density and n-body correlations in phase-space, which is essential for the cluster formation. The clusters are identified by the MST (Minimum Spanning Tree) or the SACA ('Simulated Annealing Cluster Algorithm') algorithm which finds the most-bound configuration of nucleons and clusters. Collisions among hadrons as well as Quark-Gluon-Plasma formation and parton dynamics in PHQMD are treated in the same way as in the PHSD (Parton-Hadron-String-Dynamics) transport approach. We study the time evolution of the cluster formation in the expanding medium and the stability of the clusters. We present a comparison of the PHQMD results for $d,{ }^{3} \mathrm{He}$ as well as for the hypernuclei with experimental data.
\end{abstract}

\section{Introduction}

The nature of cluster and hypernuclei production in heavy-ion collisions is one of the intriguing topics nowadays. The clusters have been observed experimentally at midrapidity from SIS up to LHC energies (cf. [1-6]). However, according to statistical models, the temperature of the surrounding matter at freeze-out is about $T \sim 100 \mathrm{MeV}$. An understanding of how weakly-bound objects, such as clusters (with binding energies below $10 \mathrm{MeV}$ ), can be formed and survive in such a hot environment appears to be a puzzle, usually called "ice in the fire".

\footnotetext{
*e-mail: E.Bratkovskaya@gsi.de
} 
The theoretical study of cluster formation - based on statistical or coalescence models can not shed much light on the microscopic origin of such phenomena. In order to understand the dynamical processes leading to the creation of clusters at midrapidity, we studied in Ref. [7] the cluster and hypernuclei formation based on the Parton-Hadron-Quantum-MolecularDynamics (PHQMD) approach [8]. The PHQMD is a n-body microscopic transport approach for the description of heavy-ion collisions including cluster production. The dynamically formed clusters are identified by the SACA and MST algorithms. The PHQMD merges the collision integrals of the Parton-Hadron-String Dynamics (PHSD) approach $[9,10]$ with density dependent 2-body potentials between baryons, similar to the Quantum Molecular Dynamics (QMD) approach. Baryons are described by Gaussian wave functions which propagate under the influence of mutual-density-dependent 2-body forces (and not due to a mean field). As demonstrated in [8], PHQMD reproduces single-particle observables for energies $\sqrt{s_{N N}} \sim 2 \div 200 \mathrm{GeV}$ and describes the cluster formation as well.

In PHQMD clusters are formed dynamically due to the potential interactions between baryons. For this study we used a Skyrme density dependent static potential and a Coulomb interaction entering the quantal Hamiltonian equations of motion with 2-body potential energies. The clusters are identified by the MST [11] or the SACA [12, 13] algorithm, which finds the most-bound configuration of nucleons and clusters. In the MST algorithm only the coordinate-space information is used to define clusters: Two nucleons are considered as part of a cluster if their distance is less than $r_{0}=4 \mathrm{fm}$ in the cluster rest system obtained by a Lorentz transformation from the computational frame. Nucleons, which are more distant, do not feel anymore an attractive interaction. The MST procedure has been recently applied to study the cluster production in different transport approaches [14]. This study showed that in QMD-like approaches, where the few-body correlations are kept, more light clusters at midrapidity are produced than in mean-field or cascade models.

In spite of the advantages of accounting for the n-body interactions (on the level of 2body potentials) compared to mean-field-based models, the semi-classical QMD models can not achieve the stability of clusters, since they cannot project the n-body density onto the quantum ground state of a cluster, which might influence the nucleon distribution inside the cluster for very late times. To overcome this problem we have fixed a cluster freeze-out time $t$ at which the cluster production is terminated: $t=t_{0} \cdot \cosh y$, where $t_{0}$ is the cluster freeze-out time at midrapidity. We call $t$ the "physical time" because it marks identical times in the rest systems. Due to time dilatation it depends on the rapidity of the cluster rest system in the center-of-mass frame of the heavy-ion collision.

\section{Cluster and hypernuclei formation in heavy-ion collisions}

Here we highlight some results from our recent study [7] and we refer the readers to this reference for details and further results. We note that the MST algorithm is applied here for cluster recognition.

We start with showing the comparison between the midrapidity clusters measured by the NA49 Collaboration in heavy-ion collisions at the SPS at CERN and the PHQMD calculations. Figure 1 shows the rapidity distribution of deuterons (left)and ${ }^{3} \mathrm{He}$ (right) for central $\mathrm{Pb}+\mathrm{Pb}$ collisions at the beam energy $E_{\text {kin }}=40 \mathrm{AGeV}$. Here we identify the clusters at $t_{0}=53 \mathrm{fm} / c$ and $67 \mathrm{fm} / c$. The shape of the rapidity distributions are well reproduced by PHQMD within the measurement uncertainties. We note also that in the whole $p_{T}$ range, where experimental data are available, the transverse-momentum spectra in different rapidity bins are remarkable well reproduced by PHQMD (cf. Fig. 9 in [7])). Similar findings hold for the AGS and RHIC BES energies where the PHQMD provides a very good description 
of the rapidity distributions, $p_{T}$ spectra and covariant coalescence function, $B_{A}$, for $d, t$, and ${ }^{3} \mathrm{He}$.
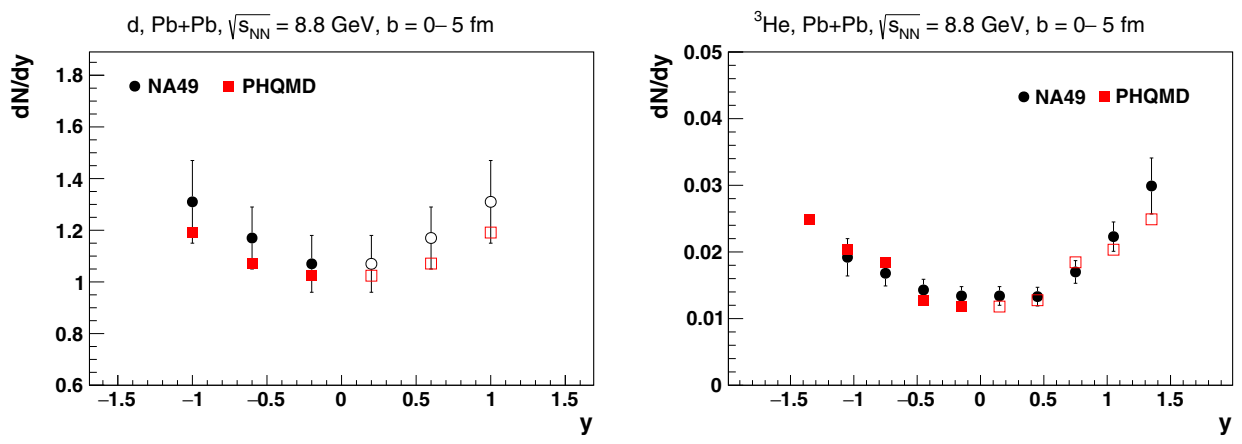

Figure 1. The rapidity distribution of deuterons (left) and ${ }^{3} \mathrm{He}$ (right) for central $\mathrm{Pb}+\mathrm{Pb}$ collisions at the beam energy $E_{k i n}=40 \mathrm{AGeV}$. The dots indicate the experimental data from the NA49 Collaboration [3]; the red squares show the PHQMD results taken at the physical time $t=t_{0} \cosh (y)$ for $t_{0}=53 \mathrm{fm} / \mathrm{c}$ for deuterons and for $t_{0}=67 \mathrm{fm} / c$ for ${ }^{3} \mathrm{He}$. The figure is taken from Ref. [7].
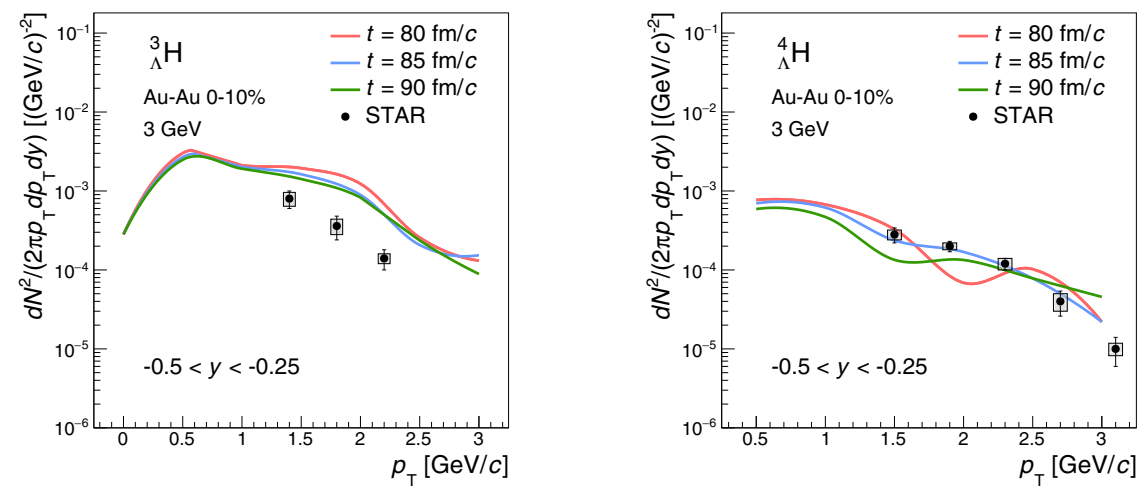

Figure 2. Transverse-momentum distribution of ${ }_{\Lambda}^{3} \mathrm{H}$ (left) and ${ }_{\Lambda}^{4} \mathrm{H}$ (right) for $-0.5<y<-0.25$ in central $\mathrm{Au}+\mathrm{Au}$ collisions at $\sqrt{s_{N N}}=3 \mathrm{GeV}$. The filled circles indicate the preliminary experimental data from the STAR Collaboration [15]. The PHQMD results are taken at the times $t=80 \mathrm{fm} / c(\mathrm{red}$ lines), $85 \mathrm{fm} / c$ (blue lines) and $90 \mathrm{fm} / c$ (green lines). The figure is taken from Ref. [7].

Recently, first preliminary data of hypernuclei production at a center-of-mass energy of $\sqrt{s_{N N}}=3 \mathrm{GeV}$ have become public. In their fixed-target program the STAR collaboration measured for two hypernuclei, ${ }_{\Lambda}^{3} \mathrm{H}$ and ${ }_{\Lambda}^{4} \mathrm{H}$, the transverse-momentum distribution $d^{2} N / p_{T} d p_{T}$ in different rapidity bins [15].

In Fig. 2 we show the results for ${ }_{\Lambda}^{3} \mathrm{H}$ (left) and ${ }_{\Lambda}^{4} \mathrm{H}$ (right) for $-0.5<y<-0.25$. The filled circles indicate the experimental data from the STAR Collaboration [15]. The PHQMD results are taken at the times $t=80 \mathrm{fm} / c$ (red lines), $85 \mathrm{fm} / c$ (blue lines) and $90 \mathrm{fm} / c$ (green lines), but at this low energy the time dependence of the cluster yield is weak. The calculations show that the trend of the experimental $p_{T}$ spectra is well reproduced. We stress that the $\Lambda N$ potential implemented in PHQMD is presently quite simple $\left(=2 / 3 V_{N N}\right)$, so we do not expect an exact quantitative agreement. We overpredict the differential yield of ${ }_{\Lambda}^{3} H$, 
but reproduce the yield of ${ }_{\Lambda}^{4} H$. This demonstrates that PHQMD in its present version is a good starting point for more sophisticated studies of hypernucleus production in this energy regime.

Since PHQMD is a microscopic transport approach and allows one to follow the history of each particle, we also studied the history of cluster production and identified when and where clusters are formed. We find a visible similarity of the cluster production at midrapidity from SIS to RHIC BES energies: the clusters are produced shortly after the collisions between the hadrons have ceased and, most important, in coordinate space they are formed behind the front of the fast expanding hadrons. This is the reason why clusters can survive. Thus, according to our PHQMD study, the "ice in the fire" puzzle can be explained by the fact that clusters come from different space regions than free nucleons. This does not exclude that they have $p_{T}$ spectra compatible with those expected from a homogeneous thermal source.

\section{Acknowledgements}

The authors acknowledge support by the Deutsche Forschungsgemeinschaft (DFG, German Research Foundation): grants BR 4000/7-1 and BL 982/3-1, by the Russian Science Foundation grant 19-42-04101 and by the GSI-IN2P3 agreement under contract number 13-70. Also we thank the COST Action THOR, CA15213 and the European Union's Horizon 2020 research and innovation program under grant agreement No 824093 (STRONG-2020). The computational resources have been provided by the LOEWE-Center for Scientific Computing and the "Green Cube" at GSI, Darmstadt.

\section{References}

[1] H.H. Gutbrod, A. Sandoval, P.J. Johansen, A.M. Poskanzer, J. Gosset, W.G. Meyer, G.D. Westfall, R. Stock, Phys. Rev. Lett. 37, 667 (1976)

[2] S. Nagamiya, M.C. Lemaire, E. Moller, S. Schnetzer, G. Shapiro, H. Steiner, I. Tanihata, Phys. Rev. C 24, 971 (1981)

[3] T. Anticic et al. (NA49), Phys. Rev. C 94, 044906 (2016), 1606.04234

[4] J. Adam et al. (STAR), Phys. Rev. C 99, 064905 (2019), 1903. 11778

[5] I. Arsene et al. (BRAHMS), Phys. Rev. C 83, 044906 (2011), 1005. 5427

[6] S. Acharya et al. (ALICE), Nucl. Phys. A 971, 1 (2018), 1710.07531

[7] S. Gläßel, V. Kireyeu, V. Voronyuk, J. Aichelin, C. Blume, E. Bratkovskaya, G. Coci, V. Kolesnikov, M. Winn, Phys. Rev. C 105, 014908 (2022), 2106.14839

[8] J. Aichelin, E. Bratkovskaya, A. Le Fèvre, V. Kireyeu, V. Kolesnikov, Y. Leifels, V. Voronyuk, G. Coci, Phys. Rev. C 101, 044905 (2020), 1907.03860

[9] W. Cassing, E.L. Bratkovskaya, Phys. Rev. C 78, 034919 (2008), 0808.0022

[10] W. Cassing, E.L. Bratkovskaya, Nucl. Phys. A 831, 215 (2009), 0907.5331

[11] J. Aichelin, Phys. Rept. 202, 233 (1991)

[12] R.K. Puri, C. Hartnack, J. Aichelin, Phys. Rev. C 54, 28 (1996)

[13] R.K. Puri, J. Aichelin, J. Comput. Phys. 162, 245 (2000), nucl-th/9811018

[14] V. Kireyeu, Phys. Rev. C 103, 054905 (2021), 2103.10542

[15] Y.H. Leung (STAR) (2021), International conference on Critical Point and Onset of Deconfinement (CPOD), https://indico.cern.ch/event/985460/ 